Brazilian Journal

of Chemical

ISSN 0104-6632

Printed in Brazil

Engineering

www.scielo.br/bjce

Vol. 35, No. 02, pp. 277 - 288, April - June, 2018

dx.doi.org/10.1590/0104-6632.20180352s20160276

\title{
CHEMICAL POTENTIALS OF HARD-CORE MOLECULES BY A STEPWISE INSERTION METHOD
}

\author{
Jéssica C. da S. L. Maciel ${ }^{1}$, Charlles R. A. Abreu ${ }^{2, *}$ and \\ Frederico W. Tavares ${ }^{1,2}$ \\ ${ }^{1}$ Programa de Engenharia Química/COPPE - Universidade Federal do Rio de Janeiro, \\ Cidade Universitária, CEP: 21.941-972, Rio de Janeiro - RJ, Brazil. \\ ${ }^{2}$ Escola de Química - Universidade Federal do Rio de Janeiro, Cidade Universitária, \\ CEP: 21.949-900, Rio de Janeiro - RJ, Brazil.
}

(Submitted: April 29, 2016 ; Revised: October 4, 2016; Accepted: November 24, 2016)

\begin{abstract}
A molecular simulation algorithm was implemented to calculate chemical potentials of hard-core molecular systems at high densities. The method is based on the Widom particle insertion method and the stepfunction character of free energy variations. The algorithm was evaluated for hard-sphere mixtures at infinite dilution approximation by varying the solute/solvent diameter ratio, for systems with reduced densities from 0.1 to 0.8 . The proposed methodology was verified by comparing simulations of trimers diluted in spheres and of single-component dimer systems with results from the literature. Then, the method was applied to mixtures of hard-spheres and dimers at several conditions regarding composition, reduced density, and bond-length/diameter ratio. The results were used to validate equations of state from the literature. The proposed approach was able to obtain accurate chemical potentials for different hard-core molecular mixtures. Lower uncertainties were obtained when comparing with traditional methods, especially at high densities.
\end{abstract}

Keywords: Chemical potential; Entropy; Hard-core potential; Monte Carlo simulation; Widom method.

\section{INTRODUCTION}

The calculation of chemical potentials by Monte Carlo simulations has already been studied and applied to several systems, mainly to systems whose particles interact by the Lennard-Jones (LJ) potential (Torrie and Valleau, 1977; Mon and Griffiths, 1985; Tej and Meredith, 2002; Virnau and Müller, 2004; Kristóf and Rutkai, 2007; Boulougouris, 2010).
For mixtures composed of simple molecules, a great number of studies and methods are available. Some of the most common methods are listed in Dietrick et al. (1989) and Kofke and Cummings (1998), which include expanded ensemble, thermodynamic integration, Bennett's method, and umbrella sampling. Nevertheless, for systems with LJ interactions, the obtained chemical potential includes both energetic and entropic contributions.

* Corresponding author: abreu@eq.ufrj.br - Phone: +55 (21) 2562-7650; jessicaclinhares@gmail.com; tavares@eq.ufrj.br

This is an extended version of the manuscript presented at the VIII Brazilian Congress of Applied Thermodynamics, CBTermo 2015, Aracaju, Brazil. 
Among the methods for chemical potential determination, the Widom test-particle insertion method (Widom, 1963) is efficient and easy to implement, but is limited to low densities and simple molecular structures (Mon and Griffiths, 1985; Tej and Meredith, 2002; Koda and Ikeda, 2002, Mehrotra et al., 2012). Under tougher conditions, however, the probability of a successful insertion becomes very low and the sampling tends to be poor. To overcome this situation, a considerable computational effort is required. Different methods have been proposed to alleviate these issues (Dietrick et al., 1989; Labík and Smith, 1994; Labik et al., 1995; Labik et al., 1998; Fay et al., 1995; Kofke and Cummings, 1998; Koda and Ikeda, 2002; Mehrotra et al., 2012). With the same purpose, in this work we aim at calculating purely entropic chemical potentials (hard-core interactions) in a way that can be applied to a wide range of densities and complexities of molecular structures. These simulations of hard-core systems are important in the validation of chemical potential calculation methods (Allen and Tildesley ,1987; Escobedo and de Pablo, 1995; Tej and Meredith, 2002). Besides this, the purely entropic chemical potential from these simulations corresponds to the combinatorial contribution of activity coefficients, a commonly used tool in phase equilibrium studies.

The proposed change of the insertion method consists in a gradual insertion of the solute. One can find other gradual insertion methods in the literature. For instance, Mon and Griffiths (1985) gradually insert or delete the solute by turning on and off the energetic interactions in two-dimensional fluids of particles with Lennard-Jones pair interaction. In another example, Tej and Meredith (2002) applied an expanded ensemble Monte Carlo method to calculate the chemical potential of nanocolloidal particles in nanocolloid-polymer mixtures, using hard-sphere model systems. Their additional ensemble variable was the diameter of the colloidal particle taken as a hard sphere. Escobedo and de Pablo (1995) simulated hard-chain molecules in an expanded ensemble whose states varied in the chain size. They executed the state transitions by adding segments to the chain. Koda and Ikeda (2002) obtained the chemical potential of parallel hard-spherocylinders using two different gradual insertion methods to obtain the insertion probability. One of the methods performs a thickening of the initial point to a sphere before lengthening, while the other process thickens the test particle after lengthening. Here, we carry out the gradual insertion by scaling the solute structure proportionally until it reaches its real size in hard-core molecular systems.

\section{CHEMICAL POTENTIAL CALCULATION}

The chemical potential obtained by the Widom method for hard-core potential systems, in an ensemble with fixed volume and number of molecules, is represented by (de Souza et al., 1994; Stamatopoulou et al., 1995; Labik et al., 1995; Frenkel and Smit, 1996; Kofke and Cummings, 1998; Boulougouris et al., 1999; Boulougouris, 2010):

$\frac{\mu^{R}}{k_{b} T}=-\ln \left(p_{1}\right)$

where $k_{b}$ is the Boltzmann constant and $p_{1}$ is the probability of a successful solute insertion at randomly selected locations and orientations in the solvent medium.

Throughout this work, we use the terms solute and solvent to denote, respectively, the molecule being inserted and the set of molecules that already exist in the system. This is done even when all molecules are identical. Under these conditions, the residual chemical potential $\left(\mu^{R}\right)$ depends only on the probability of a successful insertion. This corresponds to the free energy variation of the solvent from the initial state in the absence of any solute (state ' 0 ') to the final state in the presence of the inserted molecule (state ' 1 '), as illustrated in Figure 1. A hard-sphere solvent and a fused-sphere dimer solute were used as an example. Note that the solute is never really inserted in the simulated system because the successful attempts are recorded, but they are never actually performed.

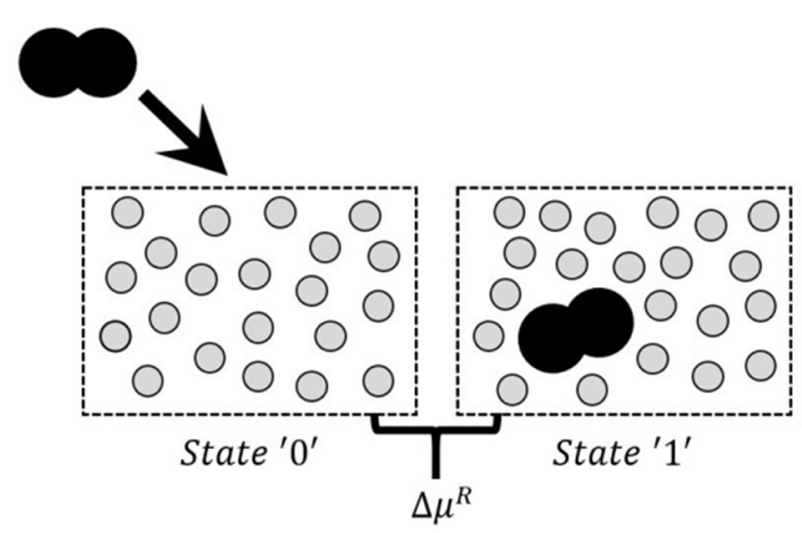

Figure 1: Illustration of the Widom method. An example of dimer insertion in hard-sphere solvent at infinite dilution. The chemical potential depends only on the probability of successful insertion attempts and corresponds to the free energy variation from state ' 0 ' to state ' 1 '. 
As mentioned before, this method is limited to low densities and to molecules with simple structures (Labik et al., 1995; Kofke and Cummings, 1998; Boulougouris et al., 1999; Boulougouris, 2010). At high densities or for systems with higher complexity, the insertion method requires a significant computational effort, that is, a large number of samples is necessary to achieve an adequate statistical sampling. In order to solve this issue, we propose here a stepwise path to the solute insertion.

The proposed path consists in inserting the molecule into the solvent in a small scale and then increasing it until the inserted solute reaches its real size. The solute structure is unaltered, in the sense that all sizes and distances vary proportionally according to a scaling factor $\lambda_{i} \in[0,1]$, in which represents a simulation step. For each transition of state from $\lambda_{i-1}$ to $\lambda_{i}$, a Monte Carlo simulation is carried out and the chemical potentials for intermediate steps are obtained. Given that thermodynamic properties depend only on the initial and final states, the total residual chemical potential will be the sum of all stepwise contributions.

Hereafter, we show that this procedure is thermodynamically consistent. Consider an athermic, N-particle system at constant volume $V$ whose potential energy $U$ consists of two terms as in:

$$
U\left(\underline{r}_{1}, \underline{r}_{2}, \ldots, \lambda\right)=\sum_{j=2}^{N} u(1, j, \lambda)+\sum_{i=2}^{N} \sum_{j=i+1}^{N} u\left(r_{i j}\right)
$$

The second term of Equation (2) represents the interaction of all other particles amongst themselves. Here, we adopted the hard-core potential model.

The first term, presented below, corresponds to the potential energy of interaction between the molecule to be inserted (1) and all other molecules in the system $(j)$. This term depends on the coupling factor $\lambda$ such that, for $\lambda=0$, the first term is "decoupled" from the system, while for $\lambda=1$ the first term corresponds to the potential energy with the molecule completely inserted. Between these two values of $\lambda$, the potential varies continuously. This dependency is generally linear, but may be non-linear as, for example, for hard-core potentials applied here.

$$
u(1, j, \lambda)=\left\{\begin{array}{ccc}
u\left(\underline{r}_{1}, \underline{r}_{2}, \ldots, r_{N-1}\right), & \text { if } & \lambda=0 \\
u\left(\underline{r}_{1}, \underline{r}_{2}, \ldots, r_{N}\right), & \text { if } & \lambda=1
\end{array}\right.
$$

The residual chemical potential can be defined as a partial derivative of the residual Helmholtz energy (Hill, 1960) as

$$
\begin{aligned}
& \mu^{R}(T, V, N)=\left(\frac{\partial A^{R}}{\partial N}\right)_{T, V} \cong \frac{A^{R}(T, V, N)-A^{R}(T, V, N-1)}{N-(N-1)} \\
& =A^{R}(T, V, N)-A^{R}(T, V, N-1)
\end{aligned}
$$

We can obtain Equation (4) for the residual chemical potential by integrating the residual free energy:

$\frac{\mu^{R}}{k_{b} T}=\frac{\mu^{R}\left(\lambda_{m}\right)}{k_{b} T}=\frac{1}{k_{b} T} \int_{0}^{1}\left(\frac{\partial A^{R}}{\partial \lambda}\right)_{T, V, N} d \lambda$

Then, we rearrange to represent all the simulation steps of the proposed algorithm,

$\frac{\mu^{R}}{k_{b} T}=\frac{1}{k_{b} T} \int_{0}^{\lambda}\left(\frac{\partial A^{R}}{\partial \lambda}\right)_{T, Y, N} d \lambda+\frac{1}{k_{b} T} \int_{\lambda_{1}}^{\lambda_{1}}\left(\frac{\partial A^{R}}{\partial \lambda}\right)_{T, Y, N} d \lambda+\ldots+\frac{1}{k_{b} T} \int_{\lambda_{\mu-1}}^{1}\left(\frac{\partial A^{R}}{\partial \lambda}\right)_{T, Y, N} d \lambda$

Finally, one can calculate the residual chemical potential from:

$\frac{\mu^{R}}{k_{b} T}=\frac{\mu_{0 \rightarrow \lambda_{1}}^{R}}{k_{b} T}+\frac{\mu_{\lambda_{1} \rightarrow \lambda_{2}}^{R}}{k_{b} T}+\ldots+\frac{\mu_{\lambda_{m-1} \rightarrow 1}^{R}}{k_{b} T}=\sum_{i=1}^{m} \frac{\mu_{\lambda_{i-1} \rightarrow \lambda_{i}}^{R}}{k_{b} T}$

Equation (7) demonstrates that the free energy variation from state ' 0 ' to state ' 1 ' is equivalent to the sum of the free energy variations between all the intermediate states as in the methodology proposed here.

For the proposed path, the coupling factor does not directly multiply the potential energy. Actually, it represents a scale factor and multiplies both the interatomic distances and atomic radii of the rigid solute molecule. This is done while maintaining the location of the geometric center and the orientation of the molecule unaltered. For $\lambda=0$, the solute molecule does not interact with the solvent. When $\lambda=1$, the solute is completely inserted into the system. In Figure 2 we illustrate the proposed path with the example of a dimer solute being inserted in a solvent of hard spheres. 


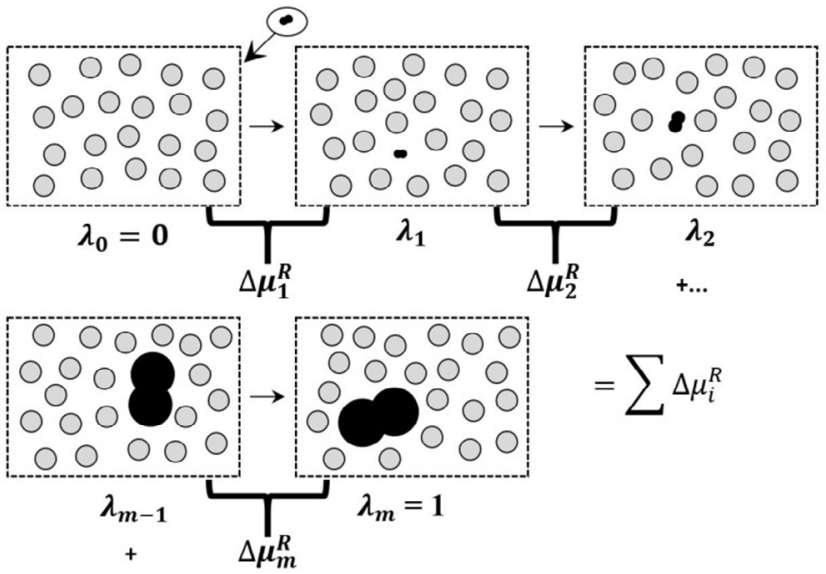

Figure 2: Illustrative scheme for the proposed gradual insertion. We present a dimer insertion in a fluid of hard sphere. Each simulation step results in a free energy increment. Initially, the residual chemical potential corresponding to the insertion of the small dimer $(\lambda)$ is calculated. Then, we obtain the residual chemical potential for each step up to the last one, with the final step $\lambda_{m}=1$, which corresponds to the solute molecule at its real size.

The method performs insertion attempts of the solute on its initial scale $\left(\lambda_{1}\right)$ to obtain the insertion chemical potential increment $\left(\Delta \mu_{1}^{R}\right)$. This procedure is identical to the conventional Widom method. The other steps intend to obtain the probabilities of increasing the solute by a pre-determined increment $\Delta \lambda_{i}$ of the scaling factor. Separate simulations carry out each step. This is an advantage because one can perform it in parallel. For these stages, the probability to increase the solute from a state $i-1$ to the next state $i$ is obtained by simulating the system with the solute already inserted with scale $\lambda_{i-1}$. This procedure consists of multiplying the full-size atomic radii and interatomic distances by $\lambda_{i}=\lambda_{i-1}+\Delta \lambda_{i}$ and recording the number of successful attempts. At the end of all simulations, the residual chemical potential between initial and final states is given by:

$$
\frac{\mu^{R}}{k_{b} T}=-\ln \left(p_{1}\right)-\sum_{i=2}^{m} \ln \left(p_{i-1, i}\right)
$$

where $p_{1}$ is the probability of a successful solute insertion attempt from $\lambda_{0}=0$ to $\lambda_{1}$ and $p_{i-1, i}$ is the probability of a successful increase of the solute size from $\lambda_{i-1}$ to $\lambda_{i}$. Even though we have shown Equation (8) for a pure component, the extension to mixtures is straightforward.

\section{MONTE CARLO SIMULATIONS}

The Monte Carlo simulations followed the Metropolis method at constant volume $(V)$ and constant number of molecules $(N)$ for athermic systems. The hard-core potential was applied in all simulated systems to obtain only entropic contributions. Thus, we calculated the free energy taking into account only the configuration because we used no attractive energetic interactions.

The volume of the simulation box was determined from the desired reduced density $\rho^{*}=\left(N_{H S} / V\right) \sigma_{s}^{3}$, where $\sigma_{s}$ is the diameter of the hard sphere which constitutes the solvent molecules, since in this work all the spheres present in the solvent have the same diameter and $N_{H S}$ is the number of hard-spheres present in the solvent. We used cubic geometry for all simulations boxes. Furthermore, we applied periodic boundary conditions in all directions. The initial configurations were generated using the software package Packmol (Martínez et al., 2009). To reduce computational effort with the overlap-test procedure, we implemented a neighbor list technique based on that of Yao et al. (2004).

We reproduced each simulation ten times independently, with the purpose of estimating the residual chemical potential uncertainties. For each stage $i$, we obtained the average of the probability and its standard deviation $(S D)$. As the chemical potential has the form $f=\mu_{i}{ }^{R} / k_{b} T=-\ln \left(p_{i}\right)$, its variance at each stage was taken as:

$S D_{\mu_{i}}^{2}=\left(-\frac{S D_{p_{i}}}{p_{i}}\right)^{2}$

where $S D_{p_{i}}$ and $S D_{\mu_{i}}$ are the probability and chemical potential standard deviations at the stage $i$, respectively.

The estimated errors for all stages lead to a propagation of uncertainty at the final chemical potential. The final chemical potential is a linear combination of the kind $f=\sum_{i=0}^{m} a_{m} A_{m}$, and because the replicas were independently measured there were no covariance and the combinatorial factor was null. Thus, we can obtain the standard deviation from

$S D_{\mu}^{2}=\sum_{i=0}^{m} S D_{\mu_{i}}^{2}$

where $S D_{\mu}$ is the final chemical potential standard deviation. 
Here, we define a cycle as a set of operations randomly proposed, performed in sequence. At each cycle, " $N$ " molecules were randomly selected from the system one at a time. For each random molecule, we proposed a random operation. Because we considered a rigid molecular structure, there were only two possible moves to be performed with the solvent molecules: rotation and translation. The translation move was applied to the geometric center, with initial position $r=\left(r_{x}, r_{y}, r_{z}\right)$, according to the procedure described in Allen and Tildesley (1987). The rotation move was applied with a randomly chosen rotation angle between $-\Delta \theta_{\text {max }}$ and $\Delta \theta_{\text {max }}$ about one of the three orthonormal axis $(\mathrm{x}, \mathrm{y}, \mathrm{z})$ also determined at random. The adopted probability of translation and rotation proposals was $50 \%$ for each. We performed these proposals for randomly selected molecules from the solvent.

In order to obtain the residual chemical potential, the first stage of the stepwise path corresponds to the Widom insertion method. The insertion of the solute molecule was attempted at each 20 cycles with $\lambda_{1}=0.1$, that means $10 \%$ of its real scale. The fixed sampling interval does not obey the detailed balance condition, but satisfies the weaker balance condition that is considered mathematically sufficient (Manousiouthakis and Deem, 1999; Ren et al., 2007; Earl and Deem, 2008; Suwa and Todo, 2010). For the insertion, we selected a uniformly distributed random position and a random orientation. Given the problems of non-uniformity and irreversibility described by Brannon et al. (2002), we applied the quaternion rotation to select a random orientation uniformly for the insertion step. This approach solves rotation problems in an uncomplicated way without the use of coordinates, allowing a more compact representation of the rotation and is free of the singularity problem (Karney, 2007).

To generate a uniform quaternion, the SHOEMAKE algorithm described by Brannon et al. (2002) was used. Then, we applied the quaternion rotation matrix of the SHOEMAKE form to the geometric center of the solute structure. With the chosen orientation, we executed an attempt to insert the solute in the random position.

In the developed algorithm, the proposed coordinates were accepted or rejected according to an overlap test (Metropolis criterion). Thus, the moves that resulted in overlaps $(\Delta U=\infty)$ were rejected. Those that did not result in overlaps $(\Delta U=0)$ were accepted. We verified the absence of overlaps by calculating the shortest distance between each atom of the chosen molecule to the other atoms in the system (considering the minimum image convention).
For the insertion step, the total number of attempted insertions of the small solute molecule $\left(\lambda_{1}=0.1\right)$ and the number of virtually accepted ones were recorded. At the end of a simulation, we used these values to obtain the insertion probability, corresponding to the first transition, according to Equation (1). The other simulations were designed to obtain the intermediate probabilities for the gradual increases.

The algorithm implemented for the particle growth was similar to the Widom method. Instead of inserting the solute into the system, the reduced molecule was already present in the solvent. The main difference lies in that we replaced the virtual insertion attempt by a virtual increase attempt for the solute. This was done by multiplying the solute structure and diameters by the corresponding scale factor. Therefore, to increase from $\lambda_{i-1}$ to $\lambda_{i}$, we multiplied the real distance of each solute atom from the geometric center of the molecule by the scale factor $\lambda_{i}$. We did the same for the diameters. To obtain the increase in probability for each state transition, we used the number of accepted moves and the total number of attempts. For this work, we adopted an increment $\Delta \lambda$ of 0.1 . We obtained the chemical potential from Equation (8).

\section{RESULTS AND DISCUSSION}

\section{Validation results}

Initially, test simulations with the original Widom method (that is, with a single insertion step) were executed so as to reproduce simulations for highly dilute solutions of spheres in spheres carried out by de Souza et al. (1994), and of dimers in spheres performed by Stamatopoulou et al. ( 1995), respectively. The successful insertion probability and chemical potential were obtained as a function of the diameter ratio, given by:

$d_{1}=\sigma_{1} / \sigma_{s}$

where $\sigma_{1}$ is the segment diameter of the solute (the largest one in the case of solutes composed of distinct spheres) and $\sigma_{\mathrm{s}}$ is the diameter of the solvent.

For sphere-in-sphere solutions, a solvent medium constituted of 108 hard spheres was simulated as in de Souza et al. (1994) for reduced density values of 0.1 , 0.4, and 0.8. Each run consisted of $10^{5}$ Monte Carlo cycles, from which the first $10^{4}$ cycles were discarded (that is, considered as equilibration cycles). In Figure 3 , one can observe a good agreement between our 
simulation results and those from de Souza et al. (1994). The results were also compared with the residual chemical potential at infinite dilution obtained from the Boublik-Mansoori-Carnahan-Starling-Leland (BMSCL) equation of state for hard sphere mixtures (de Souza et al., 1994), which is given by:

$\frac{\mu_{i}^{R, \infty}}{k_{b} T}=2 \frac{\eta d_{1}^{3}}{(1-\eta)^{3}}+3 \frac{\eta d_{1}^{2}}{(1-\eta)^{2}}+3 \frac{\eta\left(d_{1}^{2}+d_{1}-1\right)}{1-\eta}+\left(-2 d_{1}^{3}+3 d_{1}^{2}-1\right) \ln (1-\eta)$,

in which $\eta$ represents the packing fraction, that is,

$\eta=\left(\frac{\pi}{6}\right)\left(\frac{N}{V}\right) \sigma_{s}^{3}=\frac{\pi \rho^{*}}{6}$

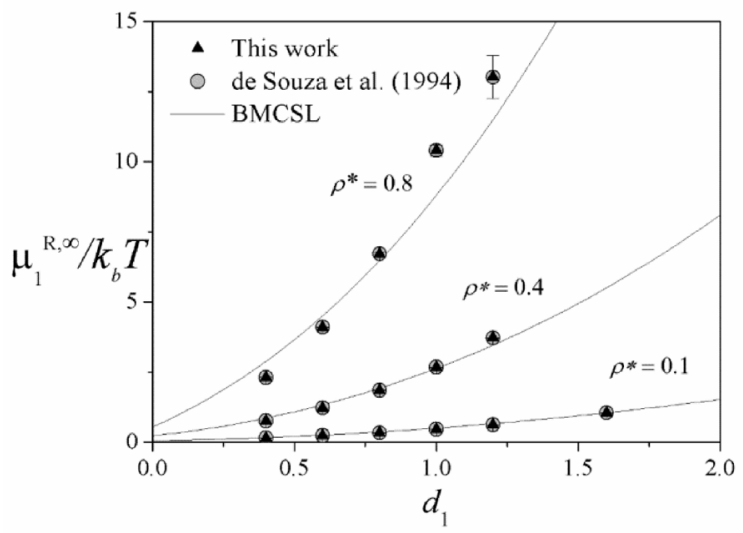

Figure 3: Residual chemical potential at infinite dilution vs. diameter ratio for hard sphere-in-sphere mixtures. The simulation results are in good agreement with the de Souza et al. (1994) and BMCSL results. This validates our single-step insertion method for hard sphere systems.

The single-step insertion of dimers in hard-sphere solvents was simulated and the results were compared to those from Stamatopoulou et al. (1995). In this case, the solute is a diatomic molecule with atomic diameters of $1.75 \AA$ and $1.20 \AA$, and a bond length of $1.27 \AA$. The reduced densities of the solvent were $0.1,0.4$, and 0.8 . For this system, $\sigma_{1}$ corresponds to the diameter of the largest sphere that composes the dimer, i.e., $\sigma_{1}=1.75 \AA$. The solvent contained 108 hard-sphere particles. Again, it can be verified in Figure 4 that a good agreement occurred between the results obtained in the present work and those from Stamatopoulou et al. (1995).

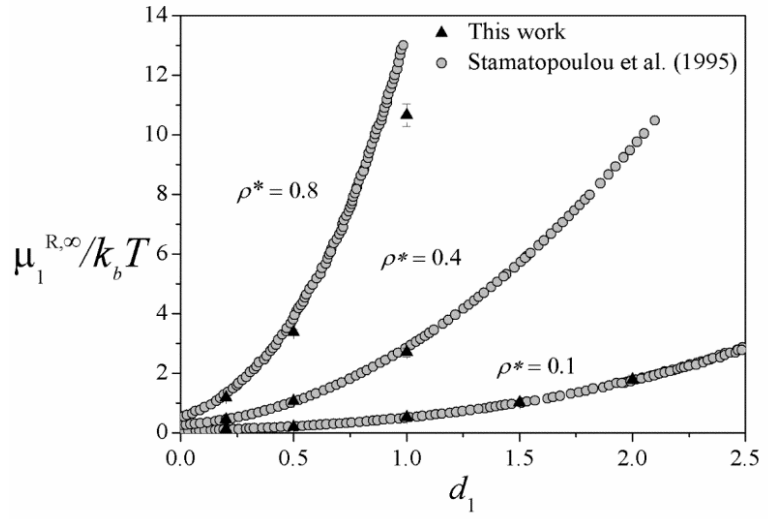

Figure 4: Probability of a successful insertion $\left(p_{1}\right)$ vs. diameter ratio $\left(d_{1}\right)$ for dimer-in-sphere mixtures at infinite dilution. The simulation results are in good agreement with the results of Stamatopoulou et al. (1995). This validates our single-step insertion method for this type of system.

We performed additional simulations by varying the density while keeping a fixed diameter ratio of 1.0 for hard-sphere systems in order to observe the density-related limitation of the Widom method. We carried out simulations at reduced densities between 0.1 and 0.9. In Figure 5, we show the results in terms of insertion probabilities to highlight the high-density problem. The insertion probabilities, shown in Figure 5a, approach zero as the density increases. This figure illustrates the increasing difficulty of inserting the solute and, consequently, of obtaining the correct probability if the original Widom method is employed. Quantitatively, the percentage deviation varied from $0.11 \%$ at $\rho^{*}=0.1$ to almost $60 \%$, at $\rho^{*}=0.9$. These values exemplify the large uncertainty in the single-step method for higher density systems, with equal number of cycles. Therefore, we can verify that such a bad sampling takes place even for the simplest systems. The insertion probability difficulties are going to be worse for large molecules. 


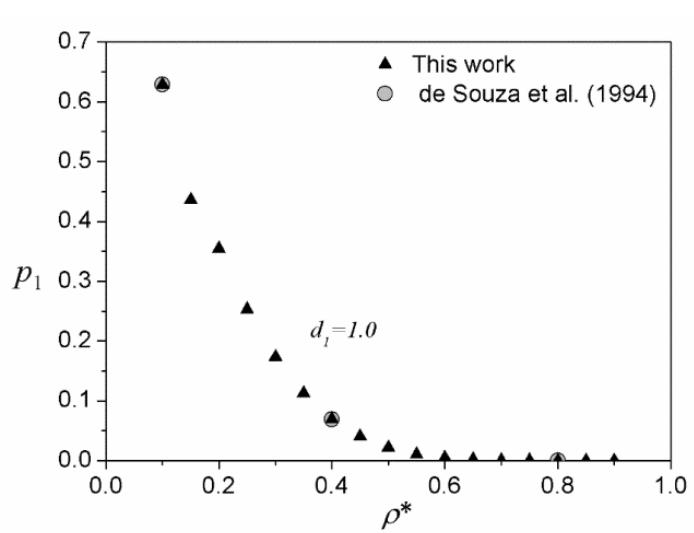

(a)

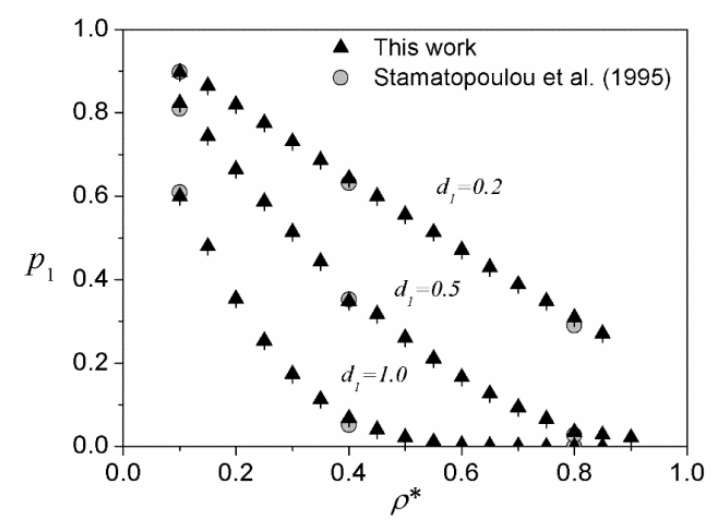

(b)

Figure 5: Probability of successful insertion vs. reduced density $\left(\rho^{*}\right)$. (a) Hard sphere mixtures at infinite dilution. (b) Dimer in hard spheres at high dilution. In both figures, we illustrate the problem of insertion at high densities: as the reduced density increases, the insertion probabilities approach zero.

One can verify the same behavior for homonuclear tangent dimers in spheres. We choose three diameter ratios, namely $0.2,0.5$, and 1.0 . The reduced density ranged from 0.1 to 0.9 . The results, shown in Figure $5 \mathrm{~b}$, illustrate a reduction in the probability of insertion with the increase of the system density for all three-diameter ratios. As expected, the lower the diameter ratio the higher is the insertion acceptance frequency for a given density. The percentage deviation for this system varied from $0.06 \%$, at $\rho^{*}=0.1$, to $0.9 \%$, at $\rho^{*}$ $=0.85$, for $d_{1}=0.2$. This small variation is related to the small diameter ratio, which does not present difficulties in the insertion process. For $d_{1}=0.5$, we have deviations of $0.15 \%$ at $\rho^{*}=0.1$ and of $8.37 \%$ at $\rho^{*}=$ 0.9 . Thus, we observe the insertion difficulty becoming problematic for the direct insertion method. The increase of uncertainty with the density increase becomes even larger for $d_{1}=1.0$. In this density, we have a percentage deviation of $0.15 \%$ at $\rho^{*}=0.1$ to almost $30 \%$ at $\rho^{*}=0.85$.

Once we had validated the basic algorithm with one-step movement (insertion), the proposed methodology with multiple steps can be tested, as presented hereafter. We validated the proposed stepwise insertion Monte Carlo method through the calculation of residual chemical potentials of highly dilute trimers in spheres. The trimers are composed of identical spheres whose centers form an equilateral triangle with side length equal to one spherical diameter, so that we could reproduce the results of Ben-Amotz et al. (1997). The simulation box contained 256 spheres and the diameter ratio $d_{1}=\sigma_{1} / \sigma_{S}$ varied from 0.1 to 0.9 at the reduced densities of $0.1,0.5$, and 0.8 .

Results are presented in Figure 6 and were compared with those of Ben-Amotz et al. (1997), who calculated the chemical potential with two different methodologies, both based on the Widom insertion method. One can observe an increase of residual chemical potential with the increase of the diameter ratio. Again, solutes with smaller diameter ratios require smaller free volume for successful insertions, which then lower the residual chemical potential. This effect is more important at high densities (liquid phase). From the results, we can only verify that the agreement with the literature and the calculation capacity of our method were satisfied. To compare the methods we have no exact information of the chemical potentials and the error values obtained by Ben-Amotz et al (1997).

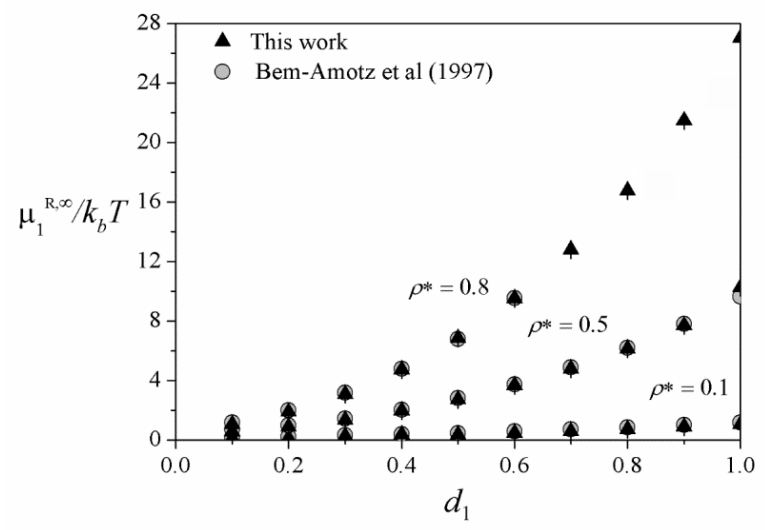

Figure 6: Chemical potential of trimers in spheres at infinite dilution vs. diameter ratio. The calculated residual chemical potentials were consistent with those from Ben-Amotz et al. (1997). Therefore, the algorithm was capable of obtaining the chemical potential for molecules with a noncolinear geometry. We observe an increase of chemical potential with the increase of the diameter ratio. 
We also applied the proposed method to systems constituted only of dimer molecules. Similar simulations were performed by Labik et al. (1995) for a homonuclear, tangent dimer solute at reduced densities of $0.5,0.6,0.7,0.8$, and 0.9 . They simulated systems with lower diameter solute and extrapolated the results to obtain the chemical potentials for higher diameters. We carried out all simulations with 600 solvent particles for this system. The results presented in Table 1 and Figure 7 show good agreement with those from Labik et al. (1995) for " $n=3 "$ (polynomial order of the extrapolation equation), considered by the authors as their more accurate results. Table 1 and Figure 7 also contain calculated residual chemical potentials from the Generalized Flory-(r'-mer) Theory (GFTheory) (Escobedo and de Pablo, 1995; Honnell and Hall, 1989) equation of state, which are also close to our values. In addition, it can be verified that the calculated errors were smaller than those from Labik et al. (1995), showing that the use of the proposed methodology can obtain reliable values for chemical potentials with lower uncertainty.

Table 1. Residual chemical potential for homonuclear, tangent dimer system.

\begin{tabular}{cccc}
\hline \multirow{2}{*}{$\boldsymbol{\rho}^{*}$} & This work & Labìk et al. (1995) & GF-Theory \\
\cline { 2 - 4 } & $\boldsymbol{\mu}^{\boldsymbol{R}, \infty} / \boldsymbol{k}_{\boldsymbol{b}} \boldsymbol{T}$ & $\boldsymbol{\mu}^{\boldsymbol{R}, \infty} \boldsymbol{k}_{\boldsymbol{b}} \boldsymbol{T}$ & $\boldsymbol{\mu}^{\boldsymbol{R}, \infty} \boldsymbol{k}_{\boldsymbol{b}} \boldsymbol{T}$ \\
\hline 0.5 & $5.86 \pm 0.005$ & $5.92 \pm 0.01$ & 5.932 \\
0.6 & $8.43 \pm 0.007$ & $8.54 \pm 0.02$ & 8.509 \\
0.7 & $11.97 \pm 0.012$ & $12.10 \pm 0.03$ & 12.048 \\
0.8 & $16.98 \pm 0.021$ & $17.13 \pm 0.05$ & 17.015 \\
0.9 & $24.21 \pm 0.033$ & $24.12 \pm 0.08$ & 24.177 \\
\hline
\end{tabular}

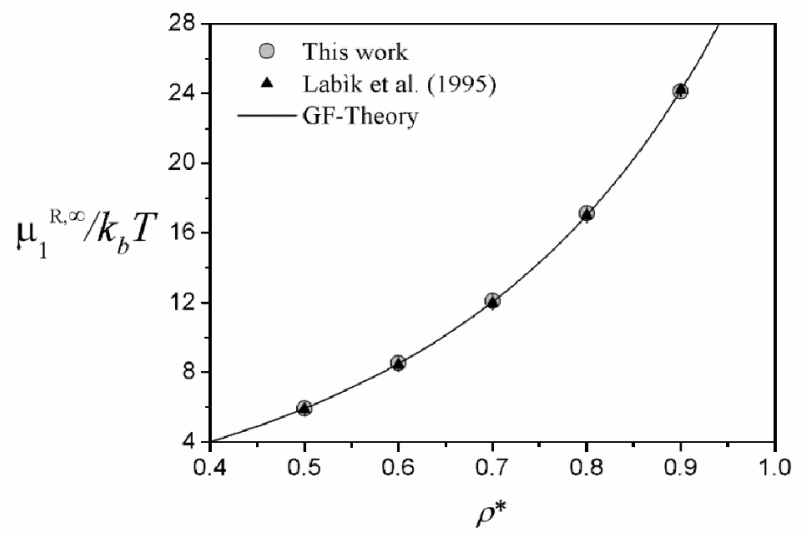

Figure 7: Chemical potential of dimers in dimers at infinite dilution vs. reduced density. The calculated residual chemical potentials were consistent with those from Labik et al. (1995) and the GF-Theory model. As expected, we observe an increase of chemical potential as the density increases.

\section{Sphere + dimer mixtures}

We applied the proposed methodology to mixtures of hard spheres (1) and dimers (2) of varying concentrations and at reduced density of mixture (defined as $\rho^{*}=\left(N_{H S_{1}} / V\right) \sigma_{1}^{3}+\left(2 N_{H S_{2}} / V\right) \sigma_{2}^{3}$, in which $\sigma_{1}=\sigma_{2}$ of 0.1 , 0.4 , and 0.8 . For all simulations, the adopted increment for the scale factor was $\Delta \lambda=0.1$. At each density, sphere+dimer mixtures with different concentrations were considered as the solvent, while either a dimer or a hard sphere was considered as the solute. Each run contained $10^{7}$ cycles, from which the 500000 initial ones were discarded, and 475000 transitions were attempted in each stage of solute growth. We tested simulation boxes with $300,500,800$, and 1200 particles to check for finite-size effects. The chosen number of particles was equal to 800 for $\rho^{*}=0.8$ and 300 for lower densities (0.4 and 0.1). Note that all dimers simulated in this mixture were homonuclear and tangent, and we assumed the diameter ratio to be unitary.

We present the obtained residual chemical potentials in Figure 8a for hard spheres and in Figure $8 \mathrm{~b}$ for dimers. The results were compared with predictions of the GF-Theory as presented by Escobedo and de Pablo (1995). For pure systems, the chemical potential of each component $\left(\mu_{i}^{\mathrm{R},}\right)$ was obtained from the integration of the equation of state, expressed in terms of compressibility factor $(Z)$ of hard chains as:

$Z=\frac{1+c_{1} \eta+c_{2} \eta^{2}+c_{3} \eta^{3}}{(1-\eta)^{3}}$

The constants used for the equation of state model were taken from Escobedo and de Pablo (1995). For a sphere (monomer), they are $c_{1}=1.0, c_{2}=1.0$, and $c_{3}=-1.0$, while for dimers they are $c_{1}=2.45696, c_{2}=$ 4.10386, and $c_{3}=-3.75503$.

For binary mixtures, an analogy to GF-Theory (Honnell and Hall, 1989; Escobedo and de Pablo, 1995) proposed by Honnell et al. (1989) was applied as a free volume correction. For a two-component system, we applied the analogy as:

$Y_{n, 1}=\frac{v_{L, 1}-v_{L, 2}}{v_{L, 2}} x_{2}$,

$Y_{n, 2}=\frac{v_{L, 1}-v_{L, 2}}{v_{L, 1}} x_{1}$, 
$\mu_{1, \text { mix }}^{R}=\left(Y_{n, 1}+1\right) \mu_{1}^{R, \circ}+Y_{n, 1} \mu_{2}^{R, \circ}$, and

$\mu_{2, \text { mix }}^{R}=\left(Y_{n, 2}+1\right) \mu_{2}^{R, \circ}+Y_{n, 1} \mu_{1}^{R, \circ}$,

where $Y_{n}$ corresponds to a correction term based on free volume differences between dimer and monomer systems, representing the free volume change with the presence of other components. The term $V_{L, 1}$ is the free volume of a system constituted only of monomers and the term $V_{L, 2}$ is the free volume of a system constituted only of dimers. The values used were $V_{L, 1}=4 / 3$ and $V_{L, 2}=9 / 4$ (Escobedo and de Pablo, 1995).

When $x_{1} \rightarrow 0$ we have that $Y_{n, 2} \rightarrow 0$, and the residual chemical potentials turn into $\mu_{2}^{R^{\circ}}$ (pure) and $\mu_{1}^{R, \infty}$ (infinite dilution). In addition, when $x_{1} \rightarrow 1$, $Y_{n, 2}$ becomes the correction for the case in which all solvent particles are replaced by hard-spheres and we have $\mu_{1}^{R^{\circ}}$ and $\mu_{2}^{R, \infty}$.

In Figure 8 one can observe good agreement between simulation results and equation-of-state calculations regarding residual chemical potentials of components 1 and 2 at mixtures with different compositions, especially at the lowest densities. As expected, the equation of state presented larger deviations at the highest density, $\rho^{*}=0.8$. However, the results followed a similar trend.

In Figure 8 the residual chemical potentials of both spheres and dimers decrease with increasing concentration of dimers. We expected this behavior because the arrangement of dimer molecules results in a greater volume of interstices due to their geometric constraint. Thus, a higher concentration of dimer molecules makes it easier to insert the solute and, consequently, lowers the residual chemical potential. Although we have simulated different size boxes, the percentage deviations presented a small variation between the different densities, compared with the single-step method. For the hard-sphere residual chemical potential, the percentage deviation was in the range of 0.083 to $0.177 \%$. For the dimer residual chemical potential, that range was from 0.02 to $0.20 \%$.

To study the effect of free volume, we calculated the residual chemical potential of dimers with different ratios of bond length/sphere diameter $\left(l / \sigma_{1}\right)$ at infinite dilution having hard spheres as solvents. In Figure 9 , the $l / \sigma$ ratio variation for homonuclear dimer particles is illustrated. Observe that, when $l / \sigma$ is zero, the dimer is reduced to a sphere, thus identical to the solvent molecules. At the other extreme $(l / \sigma=1)$, the dimer becomes a pair of tangent spheres.

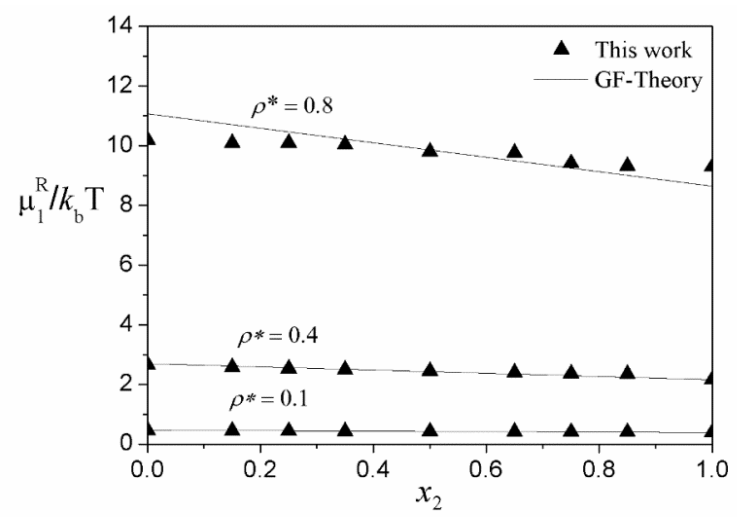

(a)

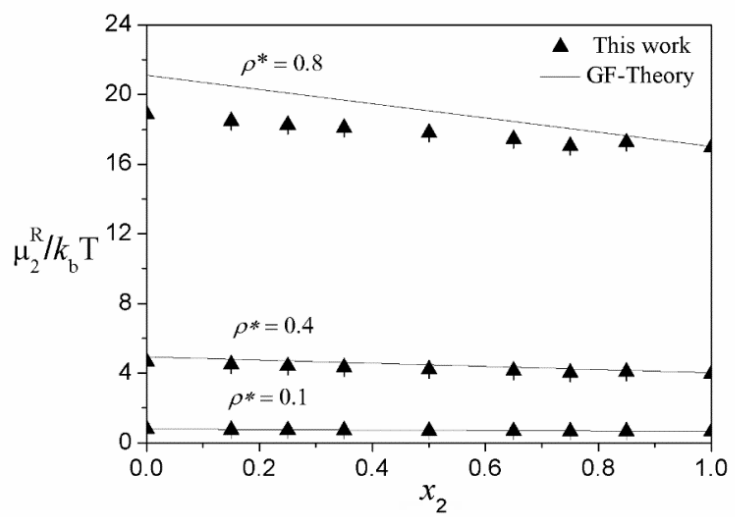

(b)

Figure 8: Residual chemical potential of solute vs. dimer fraction in the solvent, at the reduced densities of 0.1, 0.4 and 0.8. (a) Sphere as solute. (b) Dimer as solute. The residual chemical potential decreases with increasing concentration of dimers for both solutes. This behavior is consistent with the fact that a dimer molecule contains a greater volume of void space.
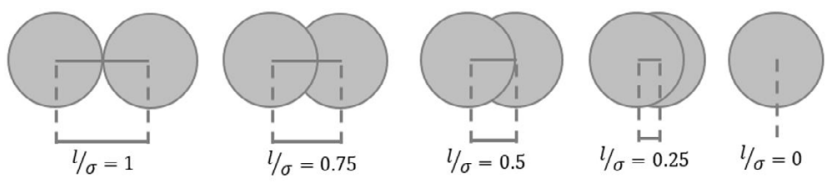

Figure 9: Representation of bond length/diameter ratio values.

In Figure 10, at low densities, there is almost no difference among the curves. This can be assigned to the largely similar solute structures and a larger interstitial space present at low densities. Nonetheless, the difference increases as the reduced density also increases. At high densities, the interstitial space becomes increasingly restricted and, accordingly, the bond length becomes an important factor for the insertion probability. For higher $l / \sigma$ ratio values, the residual chemical potential increases. Thus, a dimer with greater bond length is more difficult to insert. We observe a linear 
relationship between the chemical potential and the $l / \sigma$ ratio at all studied densities. The curve slope increases with increasing density, from 0.2334 at $\rho^{*}=0.1$ to 8.3414 at $\rho^{*}=0.8$. This quantifies the great influence of density in calculating the chemical potential in a way that makes other factors such as the bond length more influential.

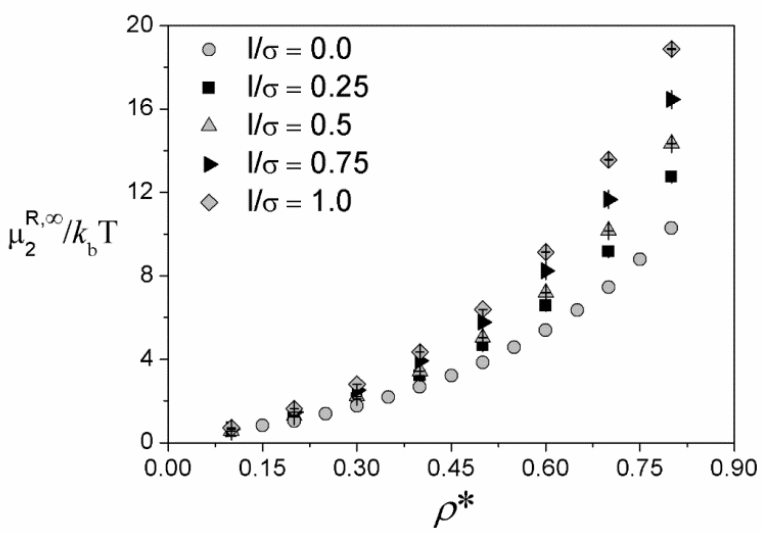

Figure 10: Residual chemical potential of dimers at infinite dilution vs. $l / \sigma$ ratio, for different reduced densities $\rho^{*}$. The influence of $l / \sigma$ on the residual chemical is linear in all densities studied.

\section{CONCLUSIONS}

The application of the Widom insertion method is a traditional way to obtain chemical potentials. However, it is limited to simple and low-density syste$\mathrm{ms}$ because its computational demand tends to become impracticable for complex molecules, especially at high densities. Based on the Widom method, we proposed an intermediate path to obtain chemical potentials of rigid molecules with hard-core potential in order to overcome the Widom method's limitation.

We validated the implemented Monte Carlo algorithm by comparing results obtained by the Widom method for sphere and dimer solutes infinitely diluted in sphere solvents. We verified the proposed methodology by comparing simulations of trimers in spheres and dimers in dimers with results from the literature. The proposed method generated similar results with lower uncertainties, especially at high densities. Then, we applied the method to systems constituted of hard-spheres and dimers. We analyzed the behavior of chemical potentials with dimer concentration to investigate the potential use of the proposed method in thermodynamics studies. The calculated residual chemical potential of each component decreases as the dimer concentration becomes higher. It corresponds to the expected behavior because dimers systems present more free space. In turn, the obtained uncertainty is promising for the obtainment of entropic residual chemical potentials even at higher densities, allowing its use in systems in the liquid state. We also obtained the residual chemical potential at infinite dilution of a dimer in hard spheres varying its bond length. We observed that the bond length had a higher influence in higher density systems.

In general, the methodology of gradual solute insertion was effective for both low and high densities for all systems studied. The calculated residual chemical potentials were consistent with the expected results. Future work will explore the range of applicability of this method to systems with increased complexity such as hard chain and real systems. In addition, the study of the applied $\lambda$ increment is necessary.

\section{REFERENCES}

Allen, M. P. and Tildesley, D. J., Computer simulation of liquids. Oxford Press, Oxford (1987).

Ben-Amotz, D., Stamatopoulou, A. and Yoon, B.J., Three-body distribution functions in hard sphere fluids. Comparison of excluded-volume-anisotropy model predictions and Monte Carlo simulation. The Journal of Chemical Physics, 107, 6831-6838 (1997).

Boulougouris, G. C., Calculation of the Chemical Potential beyond the First-Order Free-Energy Perturbation: From Deletion to Reinsertion. Journal of Chemical and Engineering Data, 55, 4140-4146 (2010).

Boulougouris, G. C., Economou, I. G. and Theodorou, D. N., On the calculation of the chemical potential using the particle deletion scheme. Molecular Physics, 96, 905-913, (1999).

Brannon, R. M., A review of useful theorems involving proper orthogonal matrices referenced to threedimensional physical space. Albuquerque, Sandia National Laboratories (2002). Available from: $<$ http://www.mech.utah.edu/ brannon/public/ rotation.pdf $>$

De Souza, L. E. S., Stamatopoulou, A. and Ben-Amotz, D., Chemical potentials of hard sphere solutes in hard sphere solvents. Monte Carlo simulations and analytical approximations. The Journal of Chemical Physics, 100, 1456-1459 (1994).

Dietrick, G. L., Scriven, L. E. and Davis, H. T., Efficient molecular simulation of chemical potentials. Journal of Chemical Physics, 90, 2370-2385 (1989). 
Earl, D. J. and Deem, M. W., Parallel Tempering : Theory, Applications, and New Perspectives. 1-21 (2008).

Escobedo, F. A. and De pablo, J. J., Chemical potential and equations of state of hard core chain molecules. The Journal of Chemical Physics, 103, 1946-1956, (1995a).

Escobedo, F. A. and De pablo, J. J,. Monte Carlo simulation of the chemical potential of polymers in an expanded ensemble. The Journal of Chemical Physics, 103, 2703-2710, (1995b).

Fay, P. J., Ray, J. R. and Wolf, R. J., Detailed balance methods for chemical potential determination. The Journal of Chemical Physics, 103, 7556, (1995).

FrenkeL, D. and Smit, B., Understanding molecular simulation From algorithms to applications. New York, Academic Press (1996).

Hill, T. L., An introduction to Statistical Thermodynamics. New York: Dover Publications, 1960.

Honnell, K. G. and Hall, C. K. A, new equation of state for athermal chains. The Journal of Chemical Physics, 90, 1841 (1989).

Karney, C. F. F., Quaternions in molecular modeling. 25, 595-604 (2007).

Koda, T. and Ikeda, S., Test of the scaled particle theory for aligned hard spherocylinders using Monte Carlo simulation. The Journal of Chemical Physics, 116, 5825 (2002).

Kofke, D. A. and Cummings, P. T. Precision and accuracy of staged free-energy perturbation methods for computing the chemical potential by molecular simulation. Fluid Phase Equilibria, 150151, 41-49, (1998).

Kristóf, T. and Rutkai, G., Chemical potential calculations by thermodynamic integration with separation shifting in adaptive sampling Monte Carlo simulations. Chemical Physics Letters, 445, 74-78 (2007).

Labìk, S., Jirásek, V., Malijevský, A. and Smith, W. R., Modifications of the SP-MC method for the computer simulation of chemical potentials: ternary mixtures of fused hard sphere fluids. Molecular Physics, 94:2, 385-393 (1998).

Labìk, S. Jirásek, V., Malijevský, A. and Smith, W. R., Computer simulation of the chemical potentials of fused hard sphere diatomic fluids. Chemical Physics Letters, 247, 227-231 (1995).

Labík, S. and Smith, W. R., Scaled Particle Theory and the Efficient Calculation of the Chemical Potential of Hard Spheres in the NVT Ensemble. Molecular Simulation, 12:1, 23-31, (1994).
Manousiouthakis, V. I. and Deem, M. W., Strict detailed balance is unnecessary in Monte Carlo simulation. The Journal of Chemical Physics, 110, 2753 (1999).

Martínez, L., Andrade, R., Brigin, E. G., Martínez J. M., PACKMOL: A Package for Building Initial Configurations for Molecular Dynamics Simulations. Journal of Computational Chemistry, 30, 2157-2164 (2009).

Mehrotra, A. S., PurI, S. and Khakhar, D. V., Field induced gradient simulations: A high throughput method for computing chemical potentials in multicomponent systems. The Journal of Chemical Physics, 136, 134108 (2012).

Mon, K. K. and Griffiths, R. B., Chemical potential by gradual insertion of a particle in Monte Carlo simulation. Physical Review A, 31, 956-959 (1985).

Ren, R., O'Keeffe, C. J. and Orkoulas, G., Sequential metropolis algorithms for fluid simulations. International Journal of Thermophysics, 28, 520 535 (2007).

Stamatopoulou, A., de Souza, L. E. S., Bem-Amotz, D. and Talbot, J., Chemical potentials of hard molecular solutes in hard sphere fluids. Monte Carlo stimulations and analytical approximations. The Journal of Chemical Physics, 102, 2109-2112 (1995).

Suwa, H. and Todo, S., Markov Chain Monte Carlo Method without Detailed Balance. Phys. Rev. Lett., $1-5$ (2010).

Tej, M. K. and Meredith, J. C., Simulation of nanocolloid chemical potentials in a hard-sphere polymer solution: Expanded ensemble Monte Carlo. The Journal of Chemical Physics, 117, 5443 (2002).

Torrie, G. M. and Valleau, J. P., Nonphysical Sampling Distributions in Monte Carlo Freeenergy Estimation: Umbrella Sampling. Journal of Computational Physics, 23, 187-199 (1977).

Virnau, P. and Müller, M., Calculation of free energy through sucessive umbrella sampling. Journal of Chemical Physics, 120, 10925 (2004).

Widom, B. Some Topics in the Theory of Fluid. Journal of Chemical Physics, 39, 2808-28012, (1963).

Yao, Z, Wang, J., Liu, G. and Cheng, M., Improved neighbor list algorithm in molecular simulations using cell decomposition and data sorting method. Computer Physics Communications, 161, 27-35, (2004). 
